\title{
Spontaneous coronary dissection and asymptomatic splenic artery aneurysms in a young woman
}

\author{
Michał Chyrchel $^{1}$, Artur Dziewierz ${ }^{1}$, Bernadeta Chyrchel $^{1}$, Tomasz Gallina², Oskar Szafrański², Andrzej Surdacki ${ }^{1}$ \\ ${ }^{1} 2^{\text {nd }}$ Department of Cardiology, Jagiellonian University Medical College, Krakow, Poland \\ ${ }^{2}$ Students' Scientific Group at the $2^{\text {nd }}$ Department of Cardiology, Jagiellonian University Medical College, Krakow, Poland
}

Adv Interv Cardiol 2020; 16, 3 (61): 340-342

DOI: https://doi.org/10.5114/aic.2020.99272

Acute coronary syndromes (ACS) remain one of the most frequent reasons for patient admissions to emergency care units. The majority of ACS are represented by myocardial infarction caused by atherosclerotic plaque rupture. Spontaneous coronary artery dissection (SCAD) remains a rare but frequently underdiagnosed cause of ACS.

A 41-year-old female patient, current smoker, without a history of previous coronary artery disease, was admitted to the emergency care unit after an episode of collapse, which was associated with chest pain. A 12-lead electrocardiogram showed an ST-segment depression in III and aVF leads. Physical examination revealed a regular heart rate of $90 \mathrm{bpm}$ and blood pressure of 165/ $90 \mathrm{~mm} \mathrm{Hg}$. The echocardiogram showed slight hypokinetic motion of the anterior and lateral wall. Baseline troponin remained within normal limits. Emergent coronary angiography revealed $50 \%$ stenosis of the left main (LM) and non-critical ostial lesion within the circumflex (CX) and left anterior descending artery (LAD) (Figure $1 \mathrm{~A}$ ). No stenosis was found within the right coronary artery (Figure $1 \mathrm{~B}$ ). At that moment the patient reported intensification of chest pain. Control angiography showed more pronounced LM stenosis, proximal occlusion of the LAD and 90\% stenosis of the proximal part of the Cx (Figure $1 \mathrm{C}$ ). Percutaneous coronary intervention $(\mathrm{PCl})$ was immediately started. After wiring, the LAD and Cx blood flow was restored and the angiographic picture was very similar to the baseline. Due to dynamic changes in coronary flow and hemodynamic instability, the patient was qualified for urgent stent implantation. Drug-eluting stents were implanted from the $L M$ to the $L A D$ and to the ostial $C X$ with the final kissing balloons' inflation (Figures $1 \mathrm{D}, \mathrm{E}$ ). Stent post-dilatation was performed within the LM with a noncompliant balloon with a good angiographic result
(Figure $1 \mathrm{~F}$ ). After the procedure, the patient was stable with blood pressure of $130 / 80 \mathrm{~mm} \mathrm{Hg}$. On the next day, echocardiography showed a preserved ejection fraction of $68 \%$, with no abnormalities. The treadmill test on the $4^{\text {th }}$ day was clinically and ECG negative. Computed tomography (CT) angiography detected relatively small splenic artery aneurysms that were qualified for conservative treatment at that stage (Figure $1 \mathrm{G}$ ). The patient was discharged home after 5 days on aspirin, ticagrelor, $\beta$-blocker, atorvastatin, and proton-pump inhibitor. No major adverse cardiac events were observed during the 1-year follow-up. Control angio-CT after 12 months confirmed the optimal result of LM bifurcation stenting (Figure $1 \mathrm{H}$ ).

Frequently, SCAD remains an underdiagnosed cause of ACS [1]. Importantly, not properly diagnosed and treated it could lead to serious clinical consequences, including cardiac arrest and death. It is based on intimal tear or intramural bleeding of the vasa vasorum leading to the creation of a false lumen and to myocardial ischemia [2]. More frequently, it appears in young or middle-aged women [3]. Coronary angiography, giving two-dimensional images, is not a perfect diagnostic modality in SCAD. Dissections, long and diffuse lesions, dye staining, and smooth-walled stenosis with an abrupt change in vessel diameter can suggest SCAD diagnosis [3, 4]. However, also unclear and non-conclusive angiographic pictures can be found. Optical coherence tomography seems to be an ideal diagnostic tool to diagnose SCAD. However, preserved coronary flow (TIMI 2 or 3 ) is mandatory to achieve conclusive imaging [5]. SCAD frequently coexists with fibromuscular dysplasia (FMD) and aneurysms [6]. CT angiography from the brain to the pelvis is recommended to exclude this pathology. Based on case reports/case series, conservative treatment with intensive 

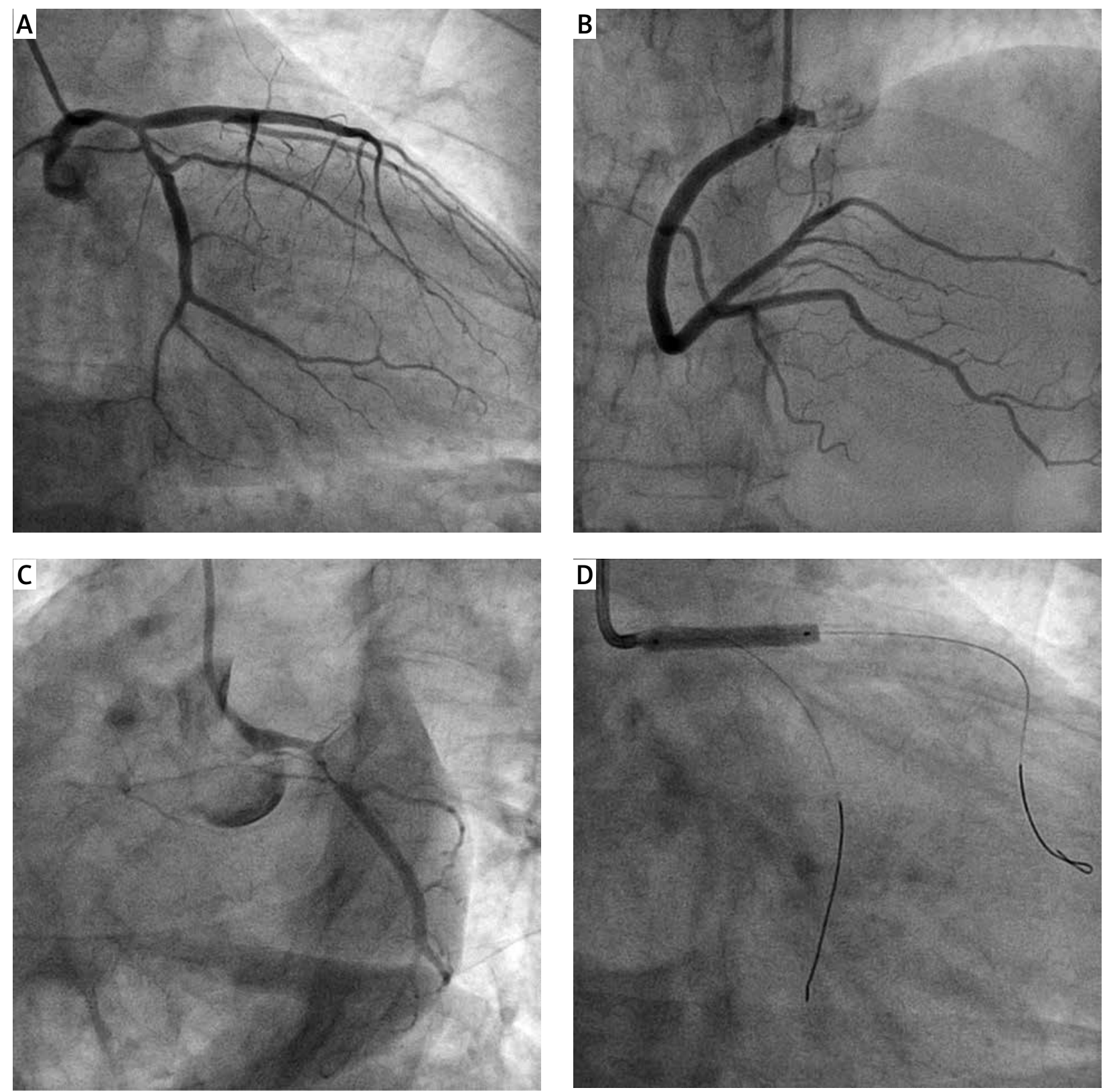

Figure 1 . Acute coronary syndrome due to spontaneous coronary dissection. A - baseline coronary angiogram of the left coronary artery, B - baseline coronary angiogram of the right coronary artery, C - control angiography at the moment of the chest pain intensification showed more pronounced left main stenosis, proximal occlusion of the left anterior descending artery and $90 \%$ stenosis of the proximal part of the circumflex artery, D - percutaneous coronary intervention procedure - direct-stenting with $3.0 \times 23 \mathrm{~mm}$ drug-eluting stent from the left main to the left anterior descending artery

pharmacotherapy is recommended in SCAD, especially in stable patients [3-5]. However, when the coronary flow is compromised, especially in high-risk subgroups (LM disease) with chest pain due to ongoing ischemia, $\mathrm{PCl}$ is necessary. In the case of long dissections associated with coronary occlusions, multi-vessel disease, or LM involvement, bypass surgery has to be considered [3]. Thus, the choice of therapeutic approach (pharmacotherapy, $\mathrm{PCl}$, or surgical intervention) should be personalized in every single clinical case. The final therapeutic decision can be supported by the European Society of Cardiology experts' statement [7].

\section{Acknowledgments}

The article processing charge was funded by the Jagiellonian University Medical College (Krakow, Poland). 

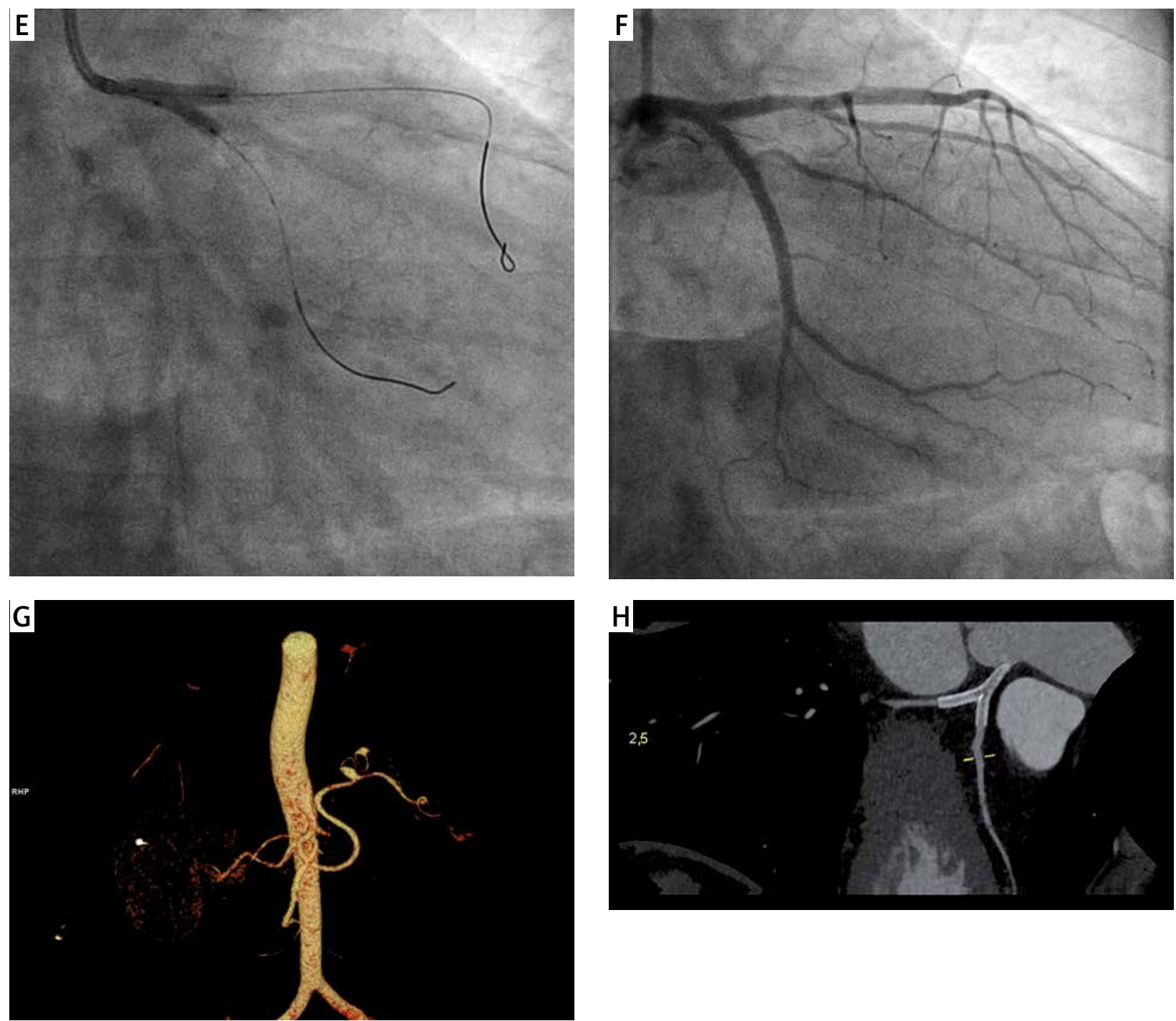

Figure 1. Cont. $\mathrm{E}$ - final kissing balloons inflation, $\mathrm{F}$ - final angiographic result, $\mathrm{G}$ - angio-CT with splenic artery aneurysms, $\mathbf{H}$ - angio-CT at 12 months with an optimal result of left main bifurcation stenting

\section{Conflict of interest}

The authors declare no conflict of interest.

\section{References}

1. Afzal A, Sarmast S, Choi JW, et al. Spontaneous coronary artery dissection: a review of pathogenesis, presentations, treatment, and outcomes. Rev Cardiovasc Med 2017; 18: 29-36.

2. Al Emam AR, Almomani A, Gilani SA, et al. Spontaneous coronary artery dissection: one disease, variable presentations, and different management approaches. Int J Angiol 2016; 25: 139-47.

3. Adlam D, Alfonso F, Maas A, Vrints C. European Society of Cardiology, acute cardiovascular care association, SCAD study group: a position paper on spontaneous coronary artery dissection. Eur Heart J 2018; 39: 3353-68.

4. Alfonso F, Paulo M, Lennie V, et al. Spontaneous coronary artery dissection: long-term follow-up of a large series of patients prospectively managed with a "conservative" therapeutic strategy. JACC Cardiovasc Interv 2012; 5: 1062-70.
5. Davlouros P, Xanthopoulou I, Tsigkas G, et al. Complete healing of spontaneous coronary artery dissection demonstrated by optical coherence tomography in a young postpartum female presenting with acute coronary syndrome. JACC Cardiovasc Interv 2017; 10: e89-90.

6. Butler R, Webster MWI, Davies G, et al. Spontaneous dissection of native coronary arteries. Heart 2005; 91: 223-4.

7. Adlam D, Alfonso F, Maas A, et al. European Society of Cardiology, acute cardiovascular care association, SCAD study group: a position paper on spontaneous coronary artery dissection. Eur Heart J 2018; 39: 3353-68. 